\title{
Mesenchymal stem cells derived secretome as an innovative cell-free therapeutic approach
}

\author{
Ejlal ABU-EL-RUB ${ }^{1,2, *}$; Ramada R. KHASAWNEH ${ }^{1}$; Fatimah A. ALMAHASNEH ${ }^{1}$; Hana M. ZEGALlaI ${ }^{3,4}$ \\ ${ }^{1}$ Department of Basic Medical Sciences, Faculty of Medicine, Yarmouk University, Irbid, 21163, Jordan \\ 2 Physiology and Pathophysiology, Faculty of Medicine, University of Manitoba, Winnipeg, R3E0W2, Canada \\ 3 Department of Pharmacology \& Therapeutics, University of Manitoba, Winnipeg, R3E0W2, Canada \\ 4 DREAM, Children's Hospital Research Institute of Manitoba, Winnipeg, R3E0W2, Canada
}

Key words: Mesencyhmal stem cells, Secretome, Immuno-modulation, Cytokines, Growth factors

\begin{abstract}
The paracrine and immunomodulatory cytokines secreted by mesenchymal stem cells (MSCs), generally referred to as the MSCs derived secretome, has substantial potential for the treatment of many chronic and degenerative diseases. MSCs secretome contains both common and disease specific cytokines and modulators that can be beneficial against a wide range of chronic diseases. Herein, we discuss the MSCs secretome composition profile and its translational applicability and the challenges surrounding its use in clinical settings.
\end{abstract}

\section{Introduction}

Over the past years, mesenchymal stem cells (MSCs) have offered new opportunities in the field of regenerative medicine through their inherited properties of multipotentiality, self-renewal, and immuno-modulation (Han et al., 2019). After being transplanted, MSCs are able to integrate with the damaged tissues and organs and adopt the required machinery to regenerate and repair the diseased tissues either by differentiating into functional cells or by secreting paracrine factors, such as anti-inflammatory and anti-apoptosis cytokines that will mitigate the presented pathology (Parekkadan and Milwid, 2010). Despite the favorable therapeutic abilities of MSCs, the percentage of engrafted cells after transplantation is usually negligible, which may affect the survival rate and long-term therapeutic potential of MSCs (Parekkadan and Milwid, 2010). Currently, researchers are sprinting in finding new approaches to enhance the engraftment percentage of MSCs through producing genetically engineered MSCs or by using different biomaterials to be combined with MSCs (Ocansey et al., 2020). Since MSCs exert their therapeutic effect by secreting paracrine factors, this led to the hypothesis that MSCs paracrine factors can be used to promote the regeneration of damaged tissues without the need to

*Address correspondence to: Ejlal Abu-El-Rub, ejlal.abuelrub@yu.edu.jo; abuelrue@myumanitoba.ca

Received: 15 July 2021; Accepted: 29 August 2021 transplant the cells themselves (Baraniak and McDevitt, 2010). The emergence of this concept was branded as "MSCs secretome", which is a cell-free cocktail of growth factors and cytokines secreted by MSCs once they are grown in certain microenvironments.

The therapeutic role of secretome in chronic and degenerative diseases

MSCs secretome contains a variety of biologically active molecules and its composition is influenced by growth conditions and environment that MSCs face (Ahangar et al., 2020). Proteomic analysis of MSCs secretome shows that it mainly contains: 1) growth factors, such as vascular endothelial growth factor (VEGF), fibroblast growth factor 2 (FGF-2), hepatocyte growth factor (HGF), and insulin-like growth factor 1 (IGF-1); 2) immune-modulatory factors, such as complement factor $\mathrm{H}$, IL-10 and indolamine-2,3dioxygenase (IDO); 3) anti-fibrotic factors, such as milk fat globule-EGF factor 8 (MFGE8), miR-150, miR-21 and miR29, and 5) Extracellular Vesicles such as exosomes, proteasomes, and free nucleic acids (Kehl et al., 2019). The MSCs derived cell-free secretome appears to be able to recapitulate many of the therapeutic properties that have been described for the MSCs themselves (Ferreira et al., 2018). Recently, the Extracellular Vesicles including the microvesicles (MVs) and exosomes which are loaded with a bunch of therapeutically effective micro-RNAs have gained special attention and validated by many pre-clinical and phase I/II clinical studies (Klyachko et al., 2020) (Fig. 1). 


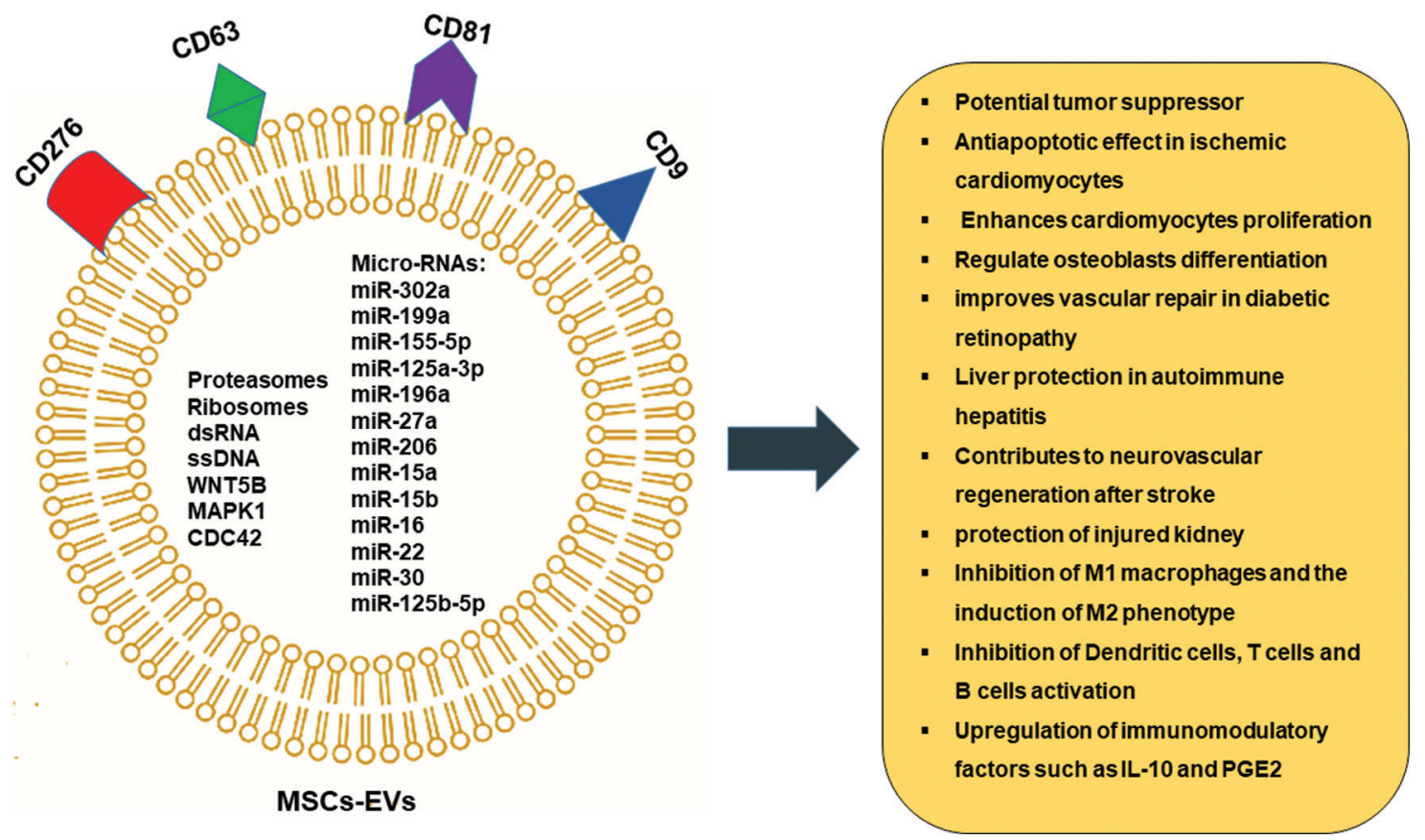

FIGURE 1. MSCs-Derived Extracellular vesicles (EVs) and its therapeutic potential.

Several studies showed that secretome can modulate and modify vital biological processes such as angiogenesis, neurogenesis, immune-modulation, wound healing, and anti-fibrotic and anti-tumour processes, which are of paramount importance for tissue repair and regeneration (Hassanzadeh et al., 2021). The regenerative and curative potential of MSCs derived secretome has been validated by many disease models (Hassanzadeh et al., 2021). Paik et al. (2020) reported that miR-150 secretome released by miR150 transfected adipose tissues-derived MSCs (AD-MSCs) abrogated the increase in systemic inflammatory cytokines associated with liver fibrosis, such as IL- 6 and TNF- $\alpha$, and it also induced the upregulation of anti-fibrotic, proliferation, and antioxidant markers in the liver. Mitchell et al showed in their study that the extracellular vesicles and nucleic acids found in the secretome derived from AD-MSCs were able to promote the regeneration of skeletal muscles following acute injury (Mitchell et al., 2019). The immunosuppressive effects of extracellular vesicles and exosomes found in MSCs derived secretome were effective in treating a wide range of autoimmune diseases, including systemic lupus erythematosus (SLE) and rheumatoid arthritis (RA) (Wang et al., 2020b). These microvesicles found in the secretome of MSCs contain different micro-RNAs, including miR-26a, miR-146 and exosomes carrying latency-associated peptide (LAP), TGF $\beta$, and thrombospondin-1 (TSP1). These compounds can suppress inflammation and excessive immune reaction against self-antigens by downregulating the serum levels of TNF- $\alpha$, IL- $1 \beta$, IL- 6 and other inflammatory cytokines. They also restrain the overstimulated activity of various immune cells including natural killer (NK) and T and B lymphocytes (Asgarpour et al., 2020).

The broad array of paracrine factors that compose the MSCs derived secretome has been corroborated to repair the damaged heart and restore normal heart function (Maghin et al., 2020) Many biological molecules in MSCs secretome are involved in the regeneration of damaged myocardium, including: 1) angiopoietin-1 (AGPT-1), cysteine rich angiogenic inducer (Cyr61), fibroblast growth factors (FGF) $1,2,4,5$ and angiogenin, which induce neovascularization and angiogenesis in the damaged myocardium; 2) neurotrophin 4 (NT 4), pentraxin 3 (PTX3 or TSG-14), bone morphogenic protein 7 (BMP 7) and stanniocalcin-1 (STC-1), which attenuate the apoptotic signaling pathway and enhance the activation of survival pathways; 3 ) secreted frizzled related protein (SFRP) 2 and 4, adrenomedullin (ADM) and hepatocyte growth factor (HGF), which reduce fibrosis and scar size, reducing remodeling; 5) Dickkopfrelated proteins (Dkk) and fibroblast growth factors (FGF) 6, 7, which stimulate the JNK signaling resulting in cardiomyogenesis and myogenesis. MSCs derived secretome also contains anti-inflammatory cytokines and immunemodulators, such as IDO, IL-10, interleukin-1 receptor antagonist (IL-1 Ra) and prostaglandin $\mathrm{E}_{2}\left(\mathrm{PGE}_{2}\right)$, which reduce inflammation and immune cell infiltration to the site of myocardial injury (Bagno et al., 2018; Gnecchi et al., 2008; Mirotsou et al., 2011).

The role of MSCs secretome in treating neurodegenerative disorders has been also reported. Different studies described MSCs secretome positive effects in Parkinson's disease (PD) by increasing viability of dopaminergic cells through the effect of $\mathrm{PGE}_{2}$. MSCs secretome preserves $\mathrm{TH}+$ neurons by increasing brain derived neurotrophic factor (BDNF) and neurotrophin-3 expression (d'Angelo et al., 2020). These neurotrophic factors are crucial for neuronal survival in the substantia nigra and promote the differentiation of dopaminergic neurons (Baquet et al., 2005). Furthermore, MSCs-derived secretome was effective in reducing $\alpha$ synuclein, nestin, and glial fibrillary acidic protein in the substantia nigra and striatum, which attenuated neuroinflammation and excessive damage to the dopaminergic cells to restore motor and cognitive performance in PD patients 
(d'Angelo et al., 2020). MSCs-derived secretome possesses the ability to stimulate neurotrophic substances such as BDNF, which is a biomarker for many neurodegenerative disorders, and promote neuronal survival pathways to counterpoise neuronal death. This makes MSCs-derived secretome a potential cure for other neurodegenerative conditions, including Alzheimer's disease and stroke (Wang et al., 2020a). Many studies have emphasized on the therapeutic potential of MSCs-derived secretome in inducing massive recovery following cerebral ischemia (Cunningham et al., 2018). The cytokines and growth factors cocktail presented in the MSCs secretome, including TGF- $\beta$, VEGF, IL-10, PDGF-AA, CXCL16 and BDNF found to inhibit neuro-inflammation, promote angiogenesis, decrease cerebral infarction and edema and inhibit the recruitment of microglial cells which all are crucial to potentiate the functional recovery (Cunningham et al., 2018). Spinal cord injury is another excruciating disorder which can benefit from MSCS-derived secretome infusion. Vawda et al found that the administration of HUC-MSCsderived secretome $48 \mathrm{~h}$ after SCI injury can reduce the lesion volume and improve the vascularity mediated by modulating the JAK/STAT and MAPK/ERK signaling pathways, and inhibit immune cell recruitment (Vawda et al., 2020). Fernandes-Cunha et al interestingly showed that delivering a lyophilized MSCs-secretome carried on viscoelastic gel composed of hyaluronic acid (HA) and chondroitin sulfate (CS) can restore the vision and promote epithelial cells proliferation which significantly reduced the scar size and hemorrhage after alkaline corneal burns (Fernandes-Cunha et al., 2019). The broad range of translational applications of MSCs-derived secretome needs further investigations to explore new therapeutic mechanisms and outcomes that can be applied to other disease models (Khatab et al., 2018; Ogata et al., 2018). A summary of the therapeutic applications of MSCs secretome is shown in Fig. 2.
Challenges and limitations surrounding the use of MSCs secretome

There are some concerns related to the use of MSCs secretome in therapeutic settings. One of the major considerations is the instability and short half-life of secretome proteins and microRNAs (Ahangar et al., 2020). Moreover, the number of MSCs that are required to produce sufficient quantities of secretome to exert equivalent regenerative effect to MSCs transplantation is about 10-25 times higher than directly administered MSCs (Ahangar et al., 2020). The heterogeneity of MSCs secretome composition is another part of the challenges regarding the clinical use of secretome (Ahangar et al., 2020; Teixeira and Salgado, 2020). The composition profile of MSCs secretome is highly influenced by the donor characteristics, the source of MSCs being isolated, and the culture and environmental conditions presented during MSCs growth and expansion (Teixeira and Salgado, 2020). Based on that, it is mandatory to standardize and optimize the secretome isolation protocol and characterize its composition profile for specific clinical applications. Moreover, there are assumed risk factors that can be associated with the use of MSCs secretome, such as diminished immune system functions following secretome administration, which may increase the risk of infection, immunodeficiency and tumor growth in treated patients (Vizoso et al., 2017). Many studies still cannot provide an updated guideline for large-scale manufacturing and production of MSCs-derived secretome and its subcomponents which is needed for clinical applications (Maumus et al., 2020).

Despite these challenges, cell-free secretome offers considerable advantages over conventional cellular therapy in terms of safety, stability and ease of handling, manipulation and storage. Further studies are recommended to discover new therapeutic applications of MSCs secretome and to find feasible approaches to increase the half-life of its
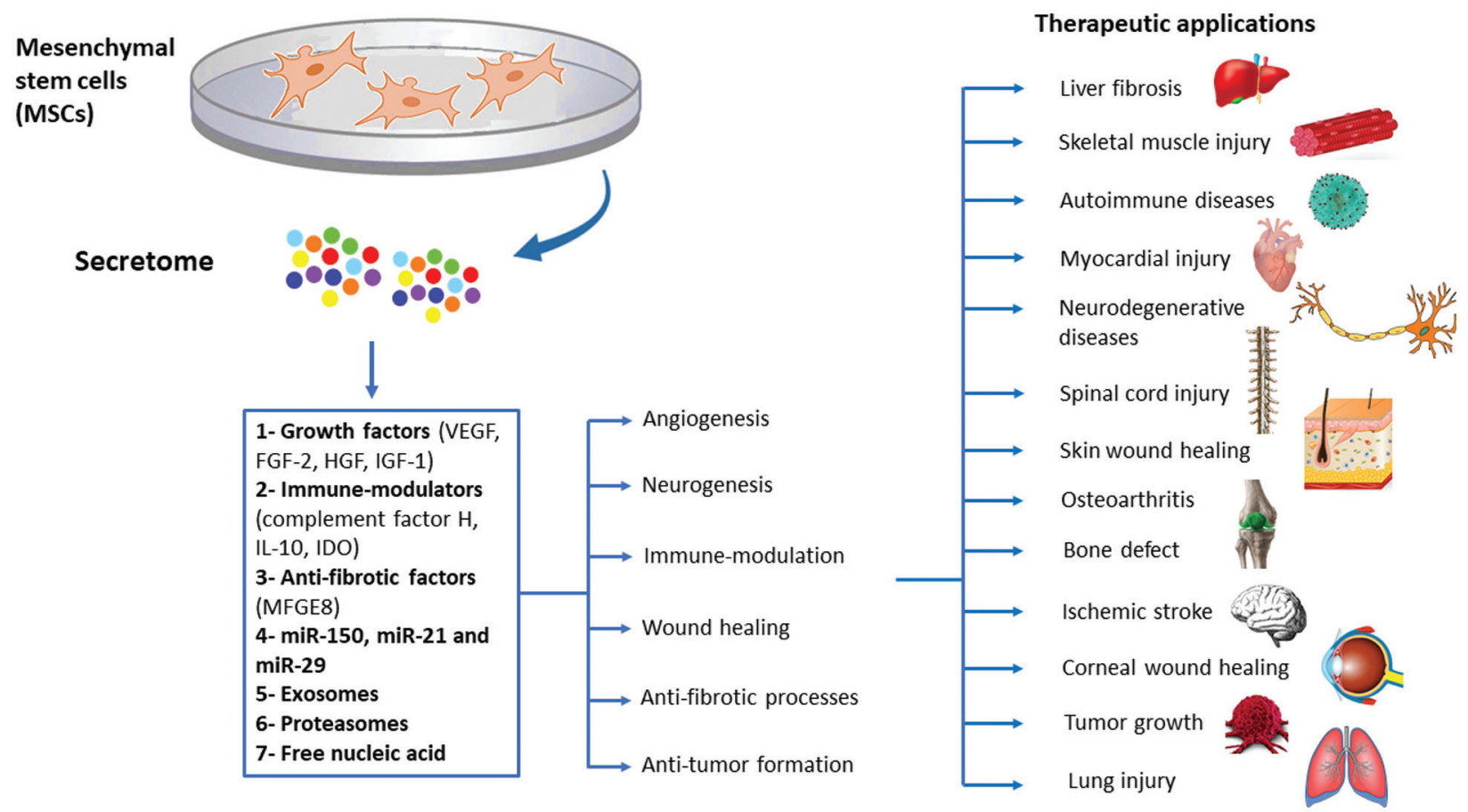

FIGURE 2. Summary of the therapeutic applications of MSCs-derived secretome. 


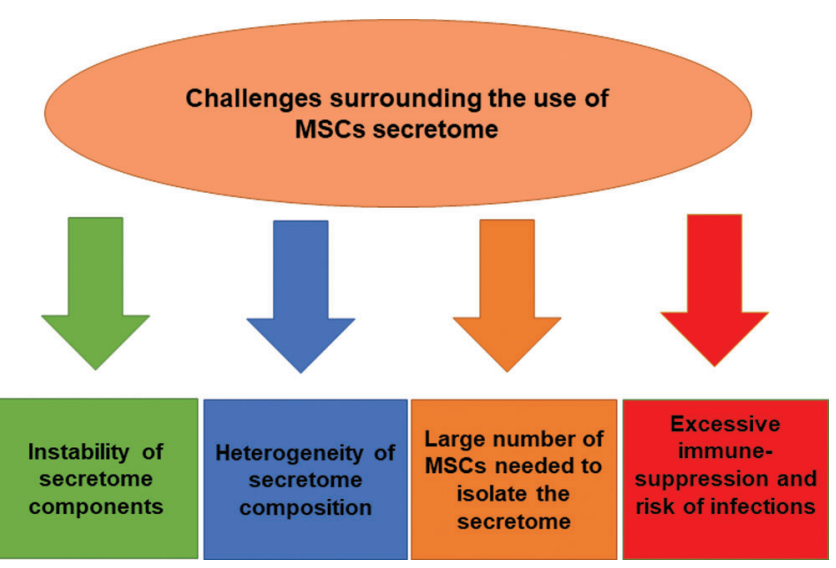

FIGURE 3. Challenges surrounding the use of MSCs-derived secretome.

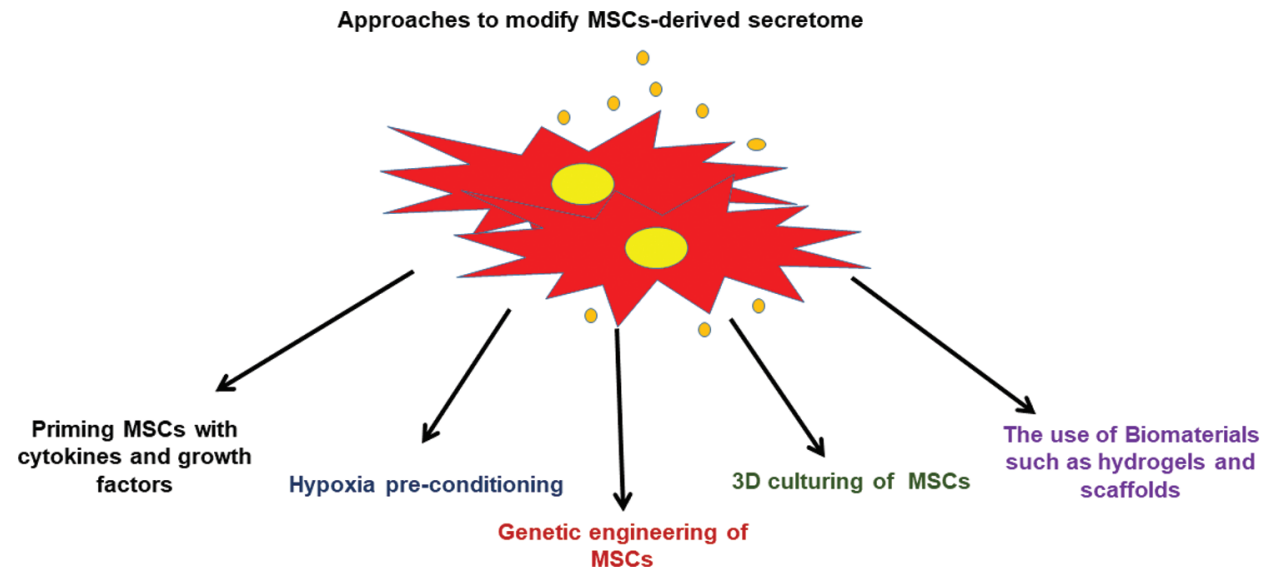

FIGURE 4. Various approaches that can be applied to modify the MSCsderived secretome.
micro-RNAs, proteins and growth factors, which can sustain and control post-infusion therapeutic effects. A graphical summary of the challenges associated with MSCs secretome' therapeutic applications can be found in Fig. 3.

Future studies should focus on using various strategies to modify the MSCs-derived secretome composition based on understanding the pathophysiological characteristics of the targeted disease which allows preparing more specific MSCs secretome that it is therapeutically effective for particular disorder. The possible approaches to modify MSCs-derived secretome have been highlighted in Fig. 4.

Authors' Contribution: Abu-El-Rub E conceptualized the content of the manuscript subtopics; Abu-El-Rub E, Khasawneh RR, Almahasneh F, Zegallai $H$ collected the literature used to write the manuscript and drafted the manuscript; Abu-El-Rub E and Almahasneh $\mathrm{F}$ revised and formatted the content of the manuscript and verified spelling, punctuation and grammatical errors; All authors have read and approved the final manuscript.

Funding Statement: The authors received no specific funding for this study.

Conflicts of Interest: The authors declare that they have no conflicts of interest to report regarding the present study.

\section{References}

Ahangar P, Mills SJ, Cowin AJ (2020). Mesenchymal stem cell secretome as an emerging cell-free alternative for improving wound repair. International Journal of Molecular Sciences 21: 7038. DOI 10.3390/ijms21197038.

Asgarpour K, Shojaei Z, Amiri F, Ai J, Mahjoubin-Tehran M, Ghasemi F, ArefNezhad R, Hamblin MR, Mirzaei H (2020). Exosomal microRNAs derived from mesenchymal stem cells: Cell-to-cell messages. Cell Communication and Signaling 18: 149. DOI 10.1186/s12964-020-00650-6.

Bagno L, Hatzistergos KE, Balkan W, Hare JM (2018). Mesenchymal stem cell-based therapy for cardiovascular disease: Progress and challenges. Molecular Therapy 26: 1610-1623. DOI 10.1016/j.ymthe.2018.05.009.

Baquet ZC, Bickford PC, Jones KR (2005). Brain-derived neurotrophic factor is required for the establishment of the proper number of dopaminergic neurons in the substantia nigra pars compacta. Journal of Neuroscience 25: 6251-6259. DOI 10.1523/JNEUROSCI.4601-04.2005.

Baraniak PR, McDevitt TC (2010). Stem cell paracrine actions and tissue regeneration. Regenerative Medicine 5: 121-143. DOI 10.2217/rme.09.74.

Cunningham CJ, Redondo-Castro E, Allan SM (2018). The therapeutic potential of the mesenchymal stem cell secretome in ischaemic stroke. Journal of Cerebral Blood Flow and Metabolism 38: 1276-1292. DOI 10.1177/0271678X18776802.

d'Angelo M, Cimini A, Castelli V (2020). Insights into the effects of mesenchymal stem cell-derived secretome in parkinson's disease. International Journal of Molecular Sciences 21: 5241. DOI 10.3390/ijms21155241.

Fernandes-Cunha GM, Na KS, Putra I, Lee HJ, Hull S, Cheng YC, Blanco IJ, Eslani M, Djalilian AR, Myung D (2019). Corneal wound healing effects of mesenchymal stem cell secretome delivered within a viscoelastic gel carrier. STEM 
CELLS Translational Medicine 8: 478-489. DOI 10.1002/ sctm.18-0178.

Ferreira JR, Teixeira GQ, Santos SG, Barbosa MA, Almeida-Porada G, Gonçalves RM (2018). Mesenchymal stromal cell secretome: influencing therapeutic potential by cellular preconditioning. Frontiers Immunology 9: 2837. DOI 10.3389/ fimmu.2018.02837.

Gnecchi M, Zhang Z, Ni A, Dzau VJ (2008). Paracrine mechanisms in adult stem cell signaling and therapy. Circulation Research 103: 1204-1219. DOI 10.1161/CIRCRESAHA.108.176826.

Han Yu, Li X, Zhang Y, Han Y, Chang F, Ding J (2019). Mesenchymal stem cells for regenerative medicine. Cells 8: 886. DOI 10.3390/cells8080886.

Hassanzadeh A, Rahman HS, Markov A, Endjun JJ, Zekiy AO et al. (2021). Mesenchymal stem/stromal cell-derived exosomes in regenerative medicine and cancer; overview of development, challenges, and opportunities. Stem Cell Research \& Therapy 12: 297. DOI 10.1186/s13287-021-02378-7.

Kehl D, Generali M, Mallone A, Heller M, Uldry AC, Cheng P, Gantenbein B, Hoerstrup SP, Weber B (2019). Proteomic analysis of human mesenchymal stromal cell secretomes: A systematic comparison of the angiogenic potential. NPJ Regenerative Medicine 4: 8. DOI 10.1038/s41536-019-0070-y.

Khatab S, Osch GV, van Kops N, Bastiaansen-Jenniskens Y, Bos PK, Verhaar J, Bernsen M, van Buul GV (2018). Mesenchymal stem cell secretome reduces pain and prevents cartilage damage in a murine osteoarthritis model. European Cells \& Materials 36: 218-230. DOI 10.22203/eCM.v036a16.

Klyachko NL, Arzt CJ, Li SM, Gololobova OA, Batrakova EV (2020). Extracellular vesicle-based therapeutics: Preclinical and clinical investigations. Pharmaceutics 12: 1171. DOI 10.3390/pharmaceutics12121171.

Maghin E, Garbati P, Quarto R, Piccoli M, Bollini S (2020). Young at heart: Combining strategies to rejuvenate endogenous mechanisms of cardiac repair. Frontiers in Bioengineering and Biotechnology 8: 447. DOI 10.3389/fbioe.2020.00447.

Maumus M, Rozier P, Boulestreau J, Jorgensen C, Noël D (2020). Mesenchymal stem cell-derived extracellular vesicles: Opportunities and challenges for clinical translation. Frontiers Bioengineering and Biotechnology 8: 997. DOI 10.3389/fbioe.2020.00997.

Mirotsou M, Jayawardena TM, Schmeckpeper J, Gnecchi M, Dzau VJ (2011). Paracrine mechanisms of stem cell reparative and regenerative actions in the heart. Journal of Molecular and Cellular Cardiology 50: 280-289. DOI 10.1016/j. yjmcc.2010.08.005.
Mitchell R, Mellows B, Sheard J, Antonioli M, Kretz O et al. (2019). Secretome of adipose-derived mesenchymal stem cells promotes skeletal muscle regeneration through synergistic action of extracellular vesicle cargo and soluble proteins. Stem Cell Research \& Therapy 10: 116. DOI 10.1186/ s13287-019-1213-1.

Ocansey DKW, Pei B, Yan Y, Qian H, Zhang X, Xu W, Mao F (2020). Improved therapeutics of modified mesenchymal stem cells: An update. Journal of Translational Medicine 18: 42. DOI 10.1186/s12967-020-02234-X.

Ogata K, Osugi M, Kawai T, Wakayama Y, Sakaguchi K, Nakamura S, Katagiri W (2018). Secretomes of mesenchymal stem cells induce early bone regeneration by accelerating migration of stem cells. Journal of Oral and Maxillofacial Surgery, Medicine, and Pathology 30: 445-451. DOI 10.1016/j. ajoms.2018.04.002.

Paik KY, Kim KH, Park JH, Lee JI, Kim OH et al. (2020). A novel antifibrotic strategy utilizing conditioned media obtained from miR-150-transfected adipose-derived stem cells: Validation of an animal model of liver fibrosis. Experimental \& Molecular Medicine 52: 438-449. DOI 10.1038/s12276-020-0393-1.

Parekkadan B, Milwid JM (2010). Mesenchymal stem cells as therapeutics. Annual Review of Biomedical Engineering 12: 87-117. DOI 10.1146/annurev-bioeng-070909-105309.

Teixeira FG, Salgado AJ (2020). Mesenchymal stem cells secretome: Current trends and future challenges. Neural Regeneration Research 15: 75-77. DOI 10.4103/1673-5374.264455.

Vawda R, Badner A, Hong J, Mikhail M, Dragas R, Xhima K, Jose A, Fehlings MG (2020). Harnessing the secretome of mesenchymal stromal cells for traumatic spinal cord injury: Multicell comparison and assessment of in vivo efficacy. Stem Cells and Development 29: 1429-1443. DOI 10.1089/ scd.2020.0079.

Vizoso FJ, Eiro N, Cid S, Schneider J, Perez-Fernandez R (2017). Mesenchymal stem cell secretome: Toward cell-free therapeutic strategies in regenerative medicine. International Journal of Molecular Sciences 18: 1852. DOI 10.3390/ijms18091852.

Wang J, Hu WW, Jiang Z, Feng MJ (2020a). Advances in treatment of neurodegenerative diseases: Perspectives for combination of stem cells with neurotrophic factors. World Journal of Stem Cells 12: 323-338. DOI 10.4252/wjsc.v12.i5.323.

Wang JH, Liu XL, Sun JM, Yang JH, Xu DH, Yan SS (2020b). Role of mesenchymal stem cell derived extracellular vesicles in autoimmunity: A systematic review. World Journal of Stem Cells 12: 879-896. DOI 10.4252/wjsc.v12.i8.879. 